\title{
Estimates on elliptic equations that hold only where the gradient is large
}

\author{
C. Imbert* and L. Silvestre ${ }^{\dagger}$
}

October 5, 2018

\begin{abstract}
We consider a function which is a viscosity solution of a uniformly elliptic equation only at those points where the gradient is large. We prove that the Hölder estimates and the Harnack inequality, as in the theory of Krylov and Safonov, apply to these functions.
\end{abstract}

\section{Introduction}

This paper is concerned with deriving estimates for functions satisfying a uniformly elliptic equation only at points where the gradient is large. For such functions, we prove a Hölder estimate together with a Harnack inequality.

Intuitively, wherever the gradient of a function $u$ is small, the function will be Lipschitz, so we should not need any further information from the equation at those points in order to obtain a Hölder regularity result. However, there is an obvious difficulty in carrying out this proof since we do not know a priori where $|\nabla u|$ will be large and where it will be small, and these sets may be very irregular. Moreover, the proofs of regularity for elliptic equations involve integral quantities in the whole domain which are hard to obtain unless the equation holds everywhere. As an extra technical difficulty, we consider viscosity solutions which are not even differentiable a priori.

The main contribution of this paper is the way the so-called $L^{\varepsilon}$ estimate is derived. We recall that deriving an $L^{\varepsilon}$ estimate consists in getting a "good" estimate on the size of the superlevel set of a nonnegative super-solution that is small at least at one point. In the uniformly elliptic case, this estimate is obtained thanks to the pointwise Alexandrov-Bakelman-Pucci estimate. Here, we proceed differently by estimating directly the measure of the set of points where the super-solution can be touched by cusps from below. This idea was inspired by [4] and [16, where a similar argument is carried out with paraboloids instead of cusps. We strongly believe that a proof based on applying the ABP estimate to the difference of the solution and a particular function, as in [5], [13] or [15], cannot be done for the result of this paper.

Main results. In order to state our results, the notion of super-solutions and sub-solutions "for large gradients" should be made precise. We do it by introducing some extremal operators depending on the ellipticity constants $\lambda$ and $\Lambda$ and also on a parameter $\gamma$ which measures how large the gradient should be. They coincide with the classical Pucci operators (plus first order terms) when $|\nabla u| \geq \gamma$, but provide no information otherwise. We will be dealing with merely (lower or upper) semi-continuous functions and their gradients together with equations will be understood in the viscosity sense. For a $C^{2}$ function $u: \Omega \subset \mathbb{R}^{d} \rightarrow$

\footnotetext{
${ }^{*}$ CNRS, UMR 8050 \& Laboratoire d'analyse et de mathématiques appliquées, Université Paris-Est Créteil Val de Marne, 61 avenue du général de Gaulle, 94010 Créteil cedex, France

$\dagger$ Mathematics Department, University of Chicago, Chicago, Illinois 60637, USA
} 
$\mathbb{R}$, we consider

$$
\begin{aligned}
& M^{+}\left(D^{2} u, \nabla u\right)= \begin{cases}\Lambda \operatorname{tr} D^{2} u^{+}-\lambda \operatorname{tr} D^{2} u^{-}+\Lambda|\nabla u| & \text { if }|\nabla u| \geq \gamma \\
+\infty & \text { otherwise }\end{cases} \\
& M^{-}\left(D^{2} u, \nabla u\right)= \begin{cases}\lambda \operatorname{tr} D^{2} u^{+}-\Lambda \operatorname{tr} D^{2} u^{-}-\Lambda|\nabla u| & \text { if }|\nabla u| \geq \gamma \\
-\infty & \text { otherwise }\end{cases}
\end{aligned}
$$

The main theorem of this paper is the following Hölder estimate.

Theorem 1.1 (Hölder estimate). For any continuous function $u: \overline{B_{1}} \rightarrow \mathbb{R}$ such that

$$
\begin{aligned}
& M^{-}\left(D^{2} u, \nabla u\right) \leq C_{0} \text { in } B_{1}, \\
& M^{+}\left(D^{2} u, \nabla u\right) \geq-C_{0} \text { in } B_{1}, \\
& \|u\|_{L^{\infty}\left(B_{1}\right)} \leq C_{0},
\end{aligned}
$$

then $u \in C^{\alpha}\left(B_{1 / 2}\right)$ and

$$
\|u\|_{C^{\alpha}\left(B_{1 / 2}\right)} \leq C C_{0}
$$

where $C$ depends on $\lambda, \Lambda$, dimension and $\gamma / C_{0}$ and $\alpha$ depends on $\lambda, \Lambda$ and dimension.

Remark 1.2. The constant $C$ in Theorem 1.1 grows like $\left(\gamma / C_{0}\right)^{\alpha}$ as $\gamma / C_{0}$ tends to $+\infty$. That is

$$
C\left(d, \lambda, \Lambda, \gamma / C_{0}\right)=\tilde{C}(d, \lambda, \Lambda)\left(1+\left(\gamma / C_{0}\right)^{\alpha}\right) .
$$

Note that when $\gamma=0$, then the constant $C$ becomes independent of $C_{0}$ and we recover the classical estimate for uniformly elliptic equations.

Our second main result is the following Harnack inequality.

Theorem 1.3 (Harnack inequality). For any non-negative continuous function $u: \overline{B_{1}} \rightarrow \mathbb{R}$ such that

$$
\begin{aligned}
& M^{-}\left(D^{2} u, \nabla u\right) \leq C_{0} \text { in } B_{1}, \\
& M^{+}\left(D^{2} u, \nabla u\right) \geq-C_{0} \text { in } B_{1},
\end{aligned}
$$

we have

$$
\sup _{B_{1 / 2}} u \leq C\left(\inf _{B_{1 / 2}} u+C_{0}\right) .
$$

The constant $C$ depends on $\lambda, \Lambda$, dimension and $\gamma /\left(C_{0}+\inf _{B_{1 / 2}} u\right)$.

We would like to emphasize that the result stated in terms of the extremal operators $M^{+}$and $M^{-}$is more general than a result which specifies equations of a particular form. A more classical way to write the assumption of Theorem (1.1) would be that for some uniformly elliptic measurable coefficients $a_{i j}(x)$, a bounded vector field $b_{j}(x)$ and a bounded function $c(x)$, the function $u$ satisfies

$$
a_{i j}(x) \partial_{i j} u+b_{i}(x) \partial_{i} u=c(x) \text { only where }|\nabla u(x)| \geq \gamma .
$$

This statement is equivalent to the assumption of our theorems if $u$ is a classical solution to the equations. Our statement with the extremal operators $M^{+}$and $M^{-}$is more adequate for the viscosity solution framework. Note also that a bounded solution to a nonlinear equation would also satisfy our assumptions if the equation is of the form

$$
F\left(D^{2} u, D u, u, x\right)=0
$$

and satisfies the conditions

1. $F(0, p, r, x) \leq C(r)|p|$ if $|p| \geq \gamma$. 
2. For every fixed $p, r$ and $x$ such that $|p| \geq \gamma, F(A, p, r, x)$ is uniformly elliptic in $A$.

In fact, in the case of classical solutions (or even $W^{2, d}$ solutions), this nonlinear situation is not more general than (1.1), since in particular we could obtain (1.1) by linearizing the equation.

As mentioned above, both Theorem 1.1 and Theorem 1.3 derive from a so-called $L^{\varepsilon}$ estimate (see Theorem 5.1). Its proof is based upon a method which seems to have originated in the work of Cabre [4 and continued in the work of Savin [16]. Such an idea has also been recently used in 2]. The idea is to estimate the measure of the super-level set of super-solutions by sliding some specific functions from below and estimating the measure of the set of contact points. In [16], and also recently in [2], the use of the ABP estimate is bypassed by sliding paraboloids from below. In [4, X. Cabre uses the distance function squared which is a natural replacement of quadratic polynomials in a Riemannian manifold. In 2], in order to prove the existence of a special barrier function to their equation (see Lemma 3.3 in [2]), they slide from below a barrier to a simpler equation. In the present paper, we slide cusps functions of the form $\varphi(x)=-|x|^{1 / 2}$.

We finally mention that we chose to state and prove results for equations with bounded (by $C_{0}$ ) right hand sides. We do so for the sake of clarity but, as the reader can check by following proofs attentively, it is possible to deal with continuous right hand side $f_{0}$ in equations and get estimates which only depend on the $L^{d}$-norm of the function $f_{0}$.

Our definition of $M^{+}$and $M^{-}$also determines the type of gradient dependence that we allow in our equations. In terms of linear equations with measurable coefficients as in (1.1), we are assuming that $b \in L^{\infty}$. In the uniformly elliptic case, the best known estimate depends only on $\|b\|_{L^{d}}$, which was obtained recently in [15. We have not yet analyzed whether we can extend our result to that kind of gradient dependence. We would also like to point out that we have not been able to obtain a satisfactory parabolic version of our results yet.

Known results. We next explain how results stated in [8, 11, 3, are related to the ones presented in this paper.

In [3, 8, a Harnack inequality is derived for solutions of some singular/degenerate equations. These solutions satisfy the assumptions of the Harnack inequality, Theorem 1.3 .

In [11, on the one hand, a Harnack inequality and Hölder estimates are proved for functions satisfying the asumptions of this article. Unfortunately, there is a gap in the proof of the lemma corresponding to the $L^{\varepsilon}$ estimate (see [11, Lemma 7]). On the other hand, an Alexandrov-Bakelman-Pucci estimate is derived in [11. The interested reader is also referred to [7, 12, 6] for other results for equations in non-divergence form and [1] for equations in divergence form that are either degenerate or singular.

In [9, an equation of the following form is studied

$$
-\operatorname{tr}\left(A(D u, u, x) D^{2} u\right)+f(x, u, D u)=0
$$

under the assumptions that

$$
\begin{array}{r}
\Lambda^{-1} \lambda(p) \mathrm{I} \leq A(x, r, p) \leq \Lambda \lambda(p) \mathrm{I}, \\
|f(x, r, p)| \leq \frac{1}{2} \Lambda(1+\lambda(p))(1+|p|)
\end{array}
$$

where $\lambda(p) \geq \lambda_{0}>0$ for $|p| \geq \gamma$. The main theorem of his paper is a Hölder continuity result, which is proved using probabilistic techniques. Note that the assumptions of our theorems contain this situation. The most important difference between the result in [9] and ours is that in that paper the equation plays some role even where $|p|$ is small, since it is important in its proof that all the eigenvalues of $A$ are comparable at every point.

Organization of the paper. The paper is organized as follows. In Section 2, we introduce tools that will be used in proofs. In Section 3, we state and prove the main new lemma. It is a measure estimate satisfied by non-negative super-solutions. In Section 5 , we deduce a so-called $L^{\varepsilon}$ estimate from the main new lemma. In Section [6, a Hölder estimate is derived from the $L^{\varepsilon}$ estimate. The last section, Section 7 is devoted to the proof of the Harnack inequality stated above. 


\section{Preliminaries}

\subsection{Scaling}

In this short subsection, we analyze how the equations involving $M^{ \pm}$change according to scaling. Those facts will be used repeatedly in Sections $4-7$

If $u$ satisfies $M^{+}\left(D^{2} u, \nabla u\right) \geq A$ in $\Omega$, then $v(x)=K u\left(x_{0}+r x\right)$ satisfies the equation $M_{r, K}^{+}\left(D^{2} v, \nabla v\right) \geq$ $K r^{2} A$ in $x_{0}+r \Omega$, where

$$
M_{r, K}^{+}\left(D^{2} v, \nabla v\right)= \begin{cases}\Lambda \operatorname{tr} D^{2} v^{+}-\lambda \operatorname{tr} D^{2} v^{-}+r \Lambda|\nabla v| & \text { if }|\nabla v| \geq r K \gamma \\ +\infty & \text { otherwise. }\end{cases}
$$

Note that if $r \leq 1$ and $K \geq 1$ then $M_{r, K}^{+} \leq M^{+}$. Therefore, in particular, $M^{+}\left(D^{2} v, \nabla v\right) \geq r^{2} K A$ in $x_{0}+r \Omega$.

Likewise, if $M^{-}\left(D^{2} u, \nabla u\right) \leq A$ in $\Omega$, then $M^{-}\left(D^{2} v, \nabla v\right) \leq r^{2} K A$ in $x_{0}+r \Omega$.

\subsection{The growing ink-spots lemma}

In this section, we state and prove a consequence of Vitali's covering lemma. This result replaces the usual Caldéron-Zygmund decomposition [5] in order to derive a so-called $L^{\varepsilon}$ estimate from Corollary 4.3] It is a statement from measure theory which is essentially the same that was used in the original work by Krylov and Safonov [13. The suggestive name growing (or crawling) ink spots was nailed by E. M. Landis according to 14 .

Lemma 2.1 (Growing ink-spots lemma). Let $E \subset F \subset B_{1}$ be two open sets. We make the following two assumptions for some constant $\delta \in(0,1)$.

- If any ball $B \subset B_{1}$ satisfies $|B \cap E|>(1-\delta)|B|$, then $B \subset F$.

- $|E| \leq(1-\delta)\left|B_{1}\right|$.

Then $|E| \leq(1-c \delta)|F|$ for some constant $c$ depending on dimension only.

Proof. For every $x \in F$, since $F$ is open, there exists some maximal ball which is contained in $F$ and contains $x$. We choose one of those balls for each $x \in F$ and call it $B^{x}$.

If $B^{x}=B_{1}$ for any $x \in F$, then the result of the theorem follows immediately since $|E| \leq(1-\delta)\left|B_{1}\right|$, so let us assume that it is not the case.

We claim that $\left|B^{x} \cap E\right| \leq(1-\delta)\left|B^{x}\right|$. Otherwise, we could find a slightly larger ball $\tilde{B}$ containing $B^{x}$ such that $|\tilde{B} \cap E|>(1-\delta)|\tilde{B}|$ and $\tilde{B} \not \subset F$, contradicting the first hypothesis.

The family of balls $B^{x}$ covers the set $F$. By the Vitali covering lemma, we can select a finite subcollection of non overlapping balls $B_{j}:=B^{x_{j}}$ such that $F \subset \bigcup_{j=1}^{K} 5 B_{j}$.

By construction, $B_{j} \subset F$ and $\left|B_{j} \cap E\right| \leq(1-\delta)\left|B_{j}\right|$. Thus, we have that $\left|B_{j} \cap F \backslash E\right| \geq \delta|B|$. Therefore

$$
\begin{aligned}
|F \backslash E| & \geq \sum_{j=1}^{K}\left|B_{j} \cap F \backslash E\right| \\
& \geq \sum_{j=1}^{K} \delta\left|B_{j}\right| \\
& =\frac{\delta}{5^{d}} \sum_{j=1}^{K}\left|5 B_{j}\right| \geq \frac{\delta}{5^{d}}|F| .
\end{aligned}
$$

The proof is finished with $c=1 / 5^{d}$. 


\section{Main new lemma}

The lemma in this section is the main difference with the classical case. It is the only lemma whose proof differs substantially with the uniformly elliptic case $(\gamma=0)$.

Lemma 3.1 (A measure estimate). There exist two small constants $\varepsilon_{0}>0$ and $\delta>0$, and a large constant $M>0$, so that if $\gamma \leq \varepsilon_{0}$, for any lower semi-continuous function $u: B_{1} \rightarrow \mathbb{R}$ such that

$$
\begin{aligned}
& u \geq 0 \text { in } B_{1}, \\
& M^{-}\left(D^{2} u, \nabla u\right) \leq 1 \text { in } B_{1}, \\
& \left|\{u>M\} \cap B_{1}\right|>(1-\delta)\left|B_{1}\right|,
\end{aligned}
$$

then $u>1$ in $B_{1 / 4}$.

Remark 3.2. Amusingly enough, the values of $M$ and $\varepsilon_{0}$ in the lemma above are absolute constants. They do not depend on $\lambda, \Lambda$ or the dimension. But the constant $\delta$ does.

\subsection{The proof for classical solutions}

The proof of Lemma 3.1 is easier to understand when $u$ is a smooth function. We will first describe the proof in this case. In the next subsection we will explain why the result holds for lower semi-continuous viscosity solutions in general.

Proposition 3.3. Lemma 3.1 holds if $u$ is a $C^{2}$ super-solution.

Proof. We do the proof by contradiction. Assume that for all $\varepsilon_{0}, \delta, M$, we can find $u$ as above and such that $u\left(x_{0}\right) \leq 1$ for some point $x_{0} \in B_{1 / 4}$.

Consider $U=\{u>M\} \cap B_{1 / 4}$. For every $x \in U$, let $y \in \overline{B_{1}}$ be a point where the minimum of $u(y)+10|y-x|^{1 / 2}$ is achieved.

On one hand, since $u \geq 0$ in $B_{1}$ and $x \in U \subset B_{1 / 4}$, then $u(z)+10|z-x|^{1 / 2}>5 \sqrt{3}$ if $z \in \partial B_{1}$. On the other hand, $u\left(x_{0}\right)+10\left|x_{0}-x\right|^{1 / 2} \leq 1+5 \sqrt{2}<5 \sqrt{3}$. Therefore, the minimum will never be achieved on the boundary and $y \in B_{1}$. Moreover, we obtain that $u(y)+10|y-x|^{1 / 2} \leq 1+5 \sqrt{2}$ and in particular $u(y) \leq 1+5 \sqrt{2}$.

We choose the constant $M$ in this lemma to be $M:=2+5 \sqrt{2}$ (note that $M$ does not depend on anything!). In this way, we know that $u(y)<M$. In particular $x \neq y$ and $|z-x|^{1 / 2}$ is differentiable at $z=x$.

Note that for one value of $x$, there could be more than one point $y$ where the minimum is achieved. However, the value of $y$ determines $x$ completely since we must have

$$
\nabla u(y)=5(x-y)|y-x|^{-3 / 2} .
$$

For convenience, let us call $\varphi(z)=-10|z|^{1 / 2}$. We thus have

$$
\begin{array}{r}
\nabla u(y)=\nabla \varphi(y-x), \\
D^{2} u(y) \geq D^{2} \varphi(y-x) .
\end{array}
$$

The expressions (3.1) and (3.2), together with the equation $M^{-}\left(D^{2} u, D u\right) \leq 1$, implies that

$$
\left|D^{2} u(y)\right| \leq C\left(1+\left|D^{2} \varphi(y-x)\right|+|\nabla \varphi(y-x)|\right)
$$

provided that $\varepsilon_{0} \leq \min _{B_{5 / 4}}|\nabla \varphi|=2 \sqrt{5}$. In the previous inequality, $C$ depends on ellipticity constants and dimension.

Since for each value of $y$, there is only one value of $x$, we can define a map $m(y):=x$. Let us call $\mathcal{T}$ the domain of the map $m$. That is $\mathcal{T}$ is the set of values that $y$ takes as $x \in U$. We know that $\mathcal{T} \subset\{y: u(y)<M\}$ and that $m(\mathcal{T})=U$.

Replacing $x=m(y)$ in (3.1) and applying the chain rule, we obtain

$$
D^{2} u(y)=D^{2} \varphi(y-m(y))(I-D m(y)) .
$$


Solving for $D m$ and using the estimate (3.3), we get (in terms of Frobenius norms)

$$
|D m(y)| \leq 1+C \frac{1+\left|D^{2} \varphi(y-x)\right|+|\varphi(y-x)|}{\left|D^{2} \varphi(y-x)\right|} \leq C .
$$

Therefore

$$
\left(1-4^{d} \delta\right)\left|B_{1 / 4}\right| \leq|U|=\int_{\mathcal{T}}|\operatorname{det} \operatorname{Dm}(y)| \mathrm{d} y \leq C|\mathcal{T}| .
$$

Since, $\mathcal{T} \subset\{y: u(y)<M\}$, from our assumptions we have that $|\mathcal{T}| \leq \delta\left|B_{1}\right|$. This is a contradicion if $\delta$ is small enough (depending on ellipticity constants and dimension). The proof is now complete.

\subsection{Formalizing the proof for viscosity solutions}

In this subsection, we explain how to derive Lemma 3.1 for merely lower semi-continuous viscosity supersolutions. In order to do so, we use classical inf-convolution techniques to reduce to the case of semiconcave viscosity super-solutions (Proposition 3.4). We then prove Lemma 3.1 in the semi-concave case (Proposition 3.5 below).

Proposition 3.4. Assume Lemma [3.1 is proved for semi-concave super-solutions. Then the lemma is also true for a lower semi-continuous super-solution $u$.

Proof. Let us consider a merely lower semi-continuous super-solution $u$ defined in $B_{1}$.

Let $v:=\min (u, 2 M)$ where $M$ is given by Lemma 3.1 for semi-concave solutions. Note that $v$ is still a super-solution because it is the minimum between two super-solutions. We have $0 \leq v \leq 2 M$.

Consider the inf-convolution of $v$ of parameter $\varepsilon>0$ :

$$
v_{\varepsilon}(x)=\inf _{y \in B_{1}}\left(v(y)+(2 \varepsilon)^{-1}|y-x|^{2}\right) .
$$

It is classical to prove that $v_{\varepsilon}$ is still a super-solution at $x \in B_{1-\delta}$ (for $\delta>0$ ) of the same equation provided that we can show that $y_{x} \notin B_{1}$.

Consider $y_{x} \in \overline{B_{1}}$ such that

$$
v_{\varepsilon}(x)=v\left(y_{x}\right)+(2 \varepsilon)^{-1}\left|y_{x}-x\right|^{2} \leq v(x) .
$$

Then

$$
\left|y_{x}-x\right| \leq 2 \sqrt{\|v\|_{\infty} \varepsilon}=2 \sqrt{2 M \varepsilon} .
$$

Thus, for any $\delta>0, v_{\varepsilon}$ is a super-solution in $B_{1-\delta}$ provided that $2 \sqrt{2 M \varepsilon}<\delta$.

Note that $v_{\varepsilon}$ is semi-concave and

$$
D^{2} v_{\varepsilon} \leq \varepsilon^{-1} I
$$

Since $v$ is lower semicontinuous, it is classical to show that $v_{\varepsilon}$ converges to $v$ in the half relaxed sense (which is exactly the same as $\Gamma$-convergence). Moreover,

$$
\{u>M\}=\bigcup_{\varepsilon>0}\left\{v_{\varepsilon}>M\right\} .
$$

Note that as $\varepsilon \rightarrow 0$, the sets $\left\{v_{\varepsilon}>M\right\}$ is an increasing nested collection, therefore

$$
|\{u>M\}|=\lim _{\varepsilon \rightarrow 0}\left|\left\{v_{\varepsilon}>M\right\}\right| .
$$

For $\varepsilon$ sufficiently small, we can apply Lemma 3.1 (appropriately scaled to the ball $B_{1-\delta}$ instead of $B_{1}$ ) and obtain that $v_{\varepsilon} \geq 1$ in $B_{(1-\delta) / 4}$. Since $u \geq v_{\varepsilon}$ and $\delta$ is arbitrarly small, the proof is finished.

Proposition 3.5. Lemma 3.1 holds if $u$ is a semi-concave viscosity super-solution. 
Proof. The main idea of the proof was already explained in Lemma 3.1 for $u \in C^{2}$. Here we need to work harder in order to deal with the technical difficulty that we do not assume the function $u$ to be second differentiable. Yet, the proof follows essentially the same lines.

In order to organize the proof. We list the main steps in bold letters.

We assume that we have a semi-concave function $u$, which satisfies

$$
u \geq 0 \quad \text { and } \quad M^{-}\left(D^{2} u, \nabla u\right) \leq 1 \text { in } B_{1} .
$$

We assume also that

$$
\min _{B_{1 / 4}} u \leq 1 \quad \text { and } \quad\left|\{u>M\} \cap B_{1}\right|>(1-\delta)\left|B_{1}\right|
$$

in order to obtain a contradiction.

\section{Step 0. Analyzing the semi-concavity assumption.}

We assume only that $D^{2} u \leq C_{0}$ in the sense that $u(x)-C_{0}|x|^{2} / 2$ is concave. This means that for every point $x_{0} \in B_{1}$ there exists a vector $p \in \mathbb{R}^{d}$ (a vector in the super-differential), which is $p=\nabla u\left(x_{0}\right)$ in case $u$ is differentiable at $x_{0}$, so that

$$
u(x) \leq u\left(x_{0}\right)+p \cdot\left(x-x_{0}\right)+\frac{C_{0}}{2}\left|x-x_{0}\right|^{2} .
$$

for all $x \in B_{1}$.

We finally recall that by Alexandrov theorem, the semi-concave function $u$ is pointwise second differentiable almost everywhere. That means that there exists a set of measure zero $E \subset B_{1}$, so that at every point $x \in B_{1} \backslash E$, the function $u$ is differentiable and there exists a symmetric matrix $D^{2} u(x)$ such that

$$
u(y)=u(x)+(y-x) \cdot \nabla u(x)+\frac{1}{2}\left\langle D^{2} u(x)(y-x),(y-x)\right\rangle+o\left(|x-y|^{2}\right) .
$$

Moreover, we also have 10

$$
\nabla u(y)=\nabla u(x)+D^{2} u(x)(y-x)+o(|x-y|),
$$

where by $\nabla u(y)$ we mean any vector in the super-differential of $u$ at $y$.

\section{Step 1. Touching $u$ with cusps from below.}

As in the proof for $u \in C^{2}$, we define $\varphi(x)=-10|x|^{1 / 2}$ and $M=2+5 \sqrt{2}$.

Consider the open set $U=\{u>M\} \cap B_{1 / 4}$. From our assumption (3.4), we have that $|U|>\left|B_{1 / 4}\right|-\delta\left|B_{1}\right|$, which is a significant measure for $\delta$ small. We can assume for example that $|U| \geq\left|B_{1 / 8}\right|$, which is a constant which depends on the dimension $d$ only.

For every $x \in U$, we look for the point $y \in B_{1}$ which realizes the following minimum.

$$
u(y)-\varphi(y-x)=\min \left\{u(z)-\varphi(z-x): z \in \overline{B_{1}}\right\} .
$$

Equivalently, if we let $q(x)=\min _{z \in \overline{B_{1}}}(u(z)-\varphi(z-x))$, we have

$$
\begin{aligned}
& u(y)=\varphi(y-x)+q(x), \\
& u(z) \geq \varphi(z-x)+q(x) \quad \forall z \in B_{1} .
\end{aligned}
$$

Since $\min _{B_{1 / 4}} u \leq 1$, we observe that $q(x) \leq 1-\min _{B_{1 / 2}} \varphi=1+5 \sqrt{2}$. Consequently, $y \notin \partial B_{1}$, since for those values of $y$ we would have $\varphi(y-x)+q(x)<0 \leq u(y)$. Moreover, $u(y)=\varphi(y-x)+q(x) \leq 1+5 \sqrt{2}=$ $M-1$. In particular $y \notin U$ and $y \neq x$.

Since $u$ is a semi-concave function, at the point $y$ where it is touched by below by the smooth function $\varphi$, it must be differentiable and $\nabla u(y)=\nabla \varphi(y-x)$. Further analysis on the second derivatives of $u$ at $y$ is postponed to later in the proof. 


\section{Step 2. Defining the contact set $\mathcal{T}$.}

We define $\mathcal{T}$ as the set of contact points $y \in \bar{B}_{1}$ for all values of $x \in U$. In other words, for any $y \in \mathcal{T}$, there exists $x_{y} \in U$ such that (3.6) holds. This definition is just a rephrasing of the definition of $\mathcal{T}$ given in the proof of Lemma 3.1.

As it was mentioned above, we have $u(y) \leq M-1$ for all $y \in \mathcal{T}$. Thus

$$
\mathcal{T} \subset B_{1} \cap\{u \leq M-1\} .
$$

\section{Step 3. $\nabla u$ is Lipschitz on $\mathcal{T}$.}

Since $u(x)>M$ for all $x \in U$ and $u(y) \leq M-1$ for all $y \in \mathcal{T}$, then we must have $|y-x|>\varepsilon$ for some $\varepsilon>0$ depending on the modulus of continuity of $u$. The function $\varphi$ has a singularity at the origin. This constant $\varepsilon>0$ tells us that we are evaluating $\varphi(y-x)$ away from this singularity where $\varphi$ is $C^{2}$ and $\left|D^{2} \varphi\right|<C \varepsilon^{-3 / 2}$.

Let $x_{1}, y_{1}$ and $x_{2}, y_{2}$ be two pairs of corresponding points (they are two pairs of $x, y$ points satisfying (3.6)). Let $r=2\left|y_{1}-y_{2}\right|$. For any $z \in B_{r}\left(y_{1}\right)$, we use the bound of $D^{2} \varphi$ above and (3.7), to obtain

$$
\begin{aligned}
u(z) & \geq \varphi\left(z-x_{1}\right) \\
& \geq \varphi\left(y_{1}-x_{1}\right)+\nabla \varphi\left(y_{1}-x_{1}\right) \cdot\left(z-y_{1}\right)-C \varepsilon^{-3 / 2} r^{2}, \\
& =u\left(y_{1}\right)+\nabla u\left(y_{1}\right) \cdot\left(z-y_{1}\right)-C \varepsilon^{-3 / 2} r^{2} .
\end{aligned}
$$

In particular, for $z=y_{2}$,

$$
u\left(y_{2}\right) \geq u\left(y_{1}\right)+\nabla u\left(y_{1}\right) \cdot\left(y_{2}-y_{1}\right)-C \varepsilon^{-3 / 2} r^{2} .
$$

Exchanging the roles of $y_{1}$ and $y_{2}$, we also get

$$
u\left(y_{1}\right) \geq u\left(y_{2}\right)+\nabla u\left(y_{2}\right) \cdot\left(y_{1}-y_{2}\right)-C \varepsilon^{-3 / 2} r^{2} .
$$

Replacing this bound for the value of $u\left(y_{1}\right)$ in the first inequality, we get

$$
u(z) \geq u\left(y_{2}\right)+\nabla u\left(y_{2}\right) \cdot\left(y_{1}-y_{2}\right)+\nabla u\left(y_{1}\right) \cdot\left(z-y_{1}\right)-C \varepsilon^{-3 / 2} r^{2} .
$$

Moreover, from (3.5), we also have

$$
u(z) \leq u\left(y_{2}\right)+\nabla u\left(y_{2}\right) \cdot\left(z-y_{2}\right)+C r^{2} .
$$

Subtracting the two inequalities above, we obtain

$$
\left(\nabla u\left(y_{1}\right)-\nabla u\left(y_{2}\right)\right) \cdot\left(z-y_{1}\right) \leq C\left(\varepsilon^{-3 / 2}+1\right) r^{2} .
$$

Since $z$ is an arbitrary point in $B_{r}\left(y_{1}\right)$, we conclude that $\left|\nabla u\left(y_{1}\right)-\nabla u\left(y_{2}\right)\right| \leq C\left(1+\varepsilon^{-3 / 2}\right) r$. That is, we proved that $\nabla u$ is Lipschitz on $\mathcal{T}$. The estimate of the Lipschitz norm $[\nabla u]_{L i p(\mathcal{T})}$ that we obtained depends on $\varepsilon$ and consequently on the modulus of continuity of $u$. It is not a universal constant.

Step 4. The map $m: \mathcal{T} \rightarrow U$.

As it was pointed out above, $u$ must be differentiable at the point $y$ and $\nabla u(y)=\nabla \varphi(y-x)$. Note that the value of $\nabla \varphi(y-x)=-5|y-x|^{-3 / 2}(y-x)$ determines uniquely the value of $(y-x)$. In particular, for every $y \in \mathcal{T}$, there is a unique $x \in U$ so that (3.6) holds, and that is the point $x$ such that $\nabla u(y)=\nabla \varphi(y-x)$. Let us define $m: \mathcal{T} \rightarrow U$ as the function that maps $y$ into $x$. That is, from the implicit definition

$$
\nabla u(y)=\nabla \varphi(y-m(y)),
$$

we deduce

$$
m(y)=y-(\nabla \varphi)^{-1} \nabla u(y),
$$

where by $(\nabla \varphi)^{-1}$ we mean the inverse of $\nabla \varphi$ as a function from $\mathbb{R}^{d} \rightarrow \mathbb{R}^{d}$.

We showed already that $\nabla u$ is Lipschitz on $\mathcal{T}$. Clearly, the map $(\nabla \varphi)^{-1}$ which maps $\nabla \varphi(y-x)$ into $y-x$ has a singularity for large gradients, or equivalently where $y-x$ is close to the origin. As it was pointed 
out above, we always have $|y-x|>\varepsilon$ for some $\varepsilon>0$ depending on the modulus of continuity of $u$. So at least we know that on the set $\mathcal{T},(\nabla \varphi)^{-1}$ will be a Lipschitz map (in fact smooth) with a Lipschitz constant depending on $\varepsilon$ (and consequently on the modulus of continuity of $u$ ). This implies that $m$ is Lipschitz. Therefore, $m$ is differentiable almost everywhere and the classical formula holds

$$
|U|=\int_{\mathcal{T}}|\operatorname{det} \operatorname{Dm}(y)| \mathrm{d} y .
$$

\section{Step 5. A universal estimate on $D m$.}

So far we have only estimated $|D m(y)|$ in terms of $\varepsilon$. This was only a technical step to justify writing the expression (3.9). Now we will obtain an estimate for $|D m(y)|$ depending only on the universal constants $\lambda, \Lambda$ and $d$.

As we mentioned in step 0 , the function $u$ is pointwise second differentiable except for a set $E$ of measure zero. In particular, for all $y \in \mathcal{T} \backslash E$, we have $M^{-}\left(D^{2} u(y), \nabla u(y)\right) \leq 1$ in the classical sense and we can do the following computations below.

We take $\gamma$ sufficiently small in order to ensure that $|\nabla u(y)|=|\nabla \varphi(y-x)|>\gamma$ for all $y \in B_{1}$ and $x \in B_{1 / 4}$. Thus, the equation $M^{-}\left(D^{2} u(y), D u(y)\right) \leq 1$ is meaningful and we obtain

$$
\lambda \operatorname{tr}\left(D^{2} u(y)\right)^{+}-\Lambda \operatorname{tr}\left(D^{2} u(y)\right)^{-}=M^{-}\left(D^{2} u(y), \nabla u(y)\right)+\Lambda|\nabla u(y)| \leq C\left(1+|y-x|^{-1 / 2}\right) .
$$

Moreover, from (3.7), we have that $D^{2} u(y) \geq D^{2} \varphi(y-x)$. In particular the negative part of the Hessian of $\varphi$ controls the Hessian of $u$ : $\left(D^{2} u(y)\right)^{-} \leq\left(D^{2} \varphi(y-x)\right)^{-}$. Combining this fact with (3.10) we obtain

$$
\left|D^{2} u(y)\right| \leq C\left(\left(D^{2} \varphi(y-x)\right)^{-}+1+|y-x|^{-1 / 2}\right) \leq C\left(1+|y-x|^{-3 / 2}\right) .
$$

We now differentiate (3.8) (recall that this is a valid computation for $y \in \mathcal{T} \backslash E$ ) and obtain

$$
D^{2} u(y)=D^{2} \varphi(y-x)(I-D m(y)) .
$$

Therefore,

$$
\begin{aligned}
|D m(y)| & =D^{2} \varphi(y-x)^{-1}\left(D^{2} \varphi(y-x)-D^{2} u(y)\right) \\
& \leq\left\|D^{2} \varphi(y-x)^{-1}\right\|\left\|D^{2} \varphi(y-x)-D^{2} u(y)\right\| \\
& \leq C
\end{aligned}
$$

where $C$ is a universal constant. For the last inequality we used that $\left\|D^{2} \varphi(y-x)^{-1}\right\|=C|x-y|^{3 / 2}$ and $\left\|D^{2} \varphi(y-x)-D^{2} u(y)\right\| \leq C\left(1+|x-y|^{-3 / 2}\right)$. Note how the dependence on $|x-y|$ cancels out. This step would not work for some other choices of $\varphi$, for example $\varphi(x)=-|x|$.

Thus, we obtained that $|D m| \leq C$ almost everywhere in $\mathcal{T}$, for a universal constant $C$. We can replace this estimate in (3.9) and obtain

$$
|U| \leq \int_{\mathcal{T}} C^{d} \mathrm{~d} y=C^{d}|\mathcal{T}|
$$

This gives us a lower bound for the measure of the set of contact points $\mathcal{T}$. Thus, $|\mathcal{T}| \geq \delta\left|B_{1}\right|$ for some $\delta>0$. Since $\mathcal{T} \subset\{u \leq M-1\}$, we obtain the contradiction with (3.4) and finish the proof.

Remark 3.6. Note that in order for the formula (3.9) to hold, we need $\nabla \Gamma$ to be Lipschitz in $\mathcal{T}$. This may be the most important difficulty of the viscosity solution adaptation of the argument.

For example, just the fact that $\Gamma$ is concave implies that $D^{2} \Gamma$ is well defined almost everywhere, but yet that does not imply the formula (3.9) since a cone functions $\Gamma(x)=|x|$ would be a counterexample for $\mathcal{T}=\{0\}$ if we understand $\nabla \Gamma$ in the sense of subdifferentials.

In this case we also have $\Gamma$ differentiable in $\mathcal{T}$. Moreover, it is not hard to see that $\Gamma$ is pointwise $C^{1,1}$ at every point $y \in \mathcal{T}$ in the sense that there exists a constant $C$ and $b \in \mathbb{R}^{d}$ such that $-C|h|^{2} \leq$ $\Gamma(y+h)-\Gamma(y)-b \cdot h \leq C|h|^{2}$. These facts allow us to differentiate (3.8) and get (3.11). 


\section{A barrier function and the doubling property}

Consider the barrier function $b(x)=|x|^{-p}$. Assume initially that $\gamma=0$. We compute, for $x \in B_{2} \backslash\{0\}$,

$$
\begin{aligned}
M^{-}\left(D^{2} b, \nabla b\right) & =\lambda p(p+1)|x|^{-p-2}-\Lambda(d-1) p|x|^{-p-2}-\Lambda p|x|^{-p-1} \\
& =p|x|^{-p-2}(\lambda(p+1)-\Lambda(d-1)-\Lambda|x|) \\
& \geq p|x|^{-p-2}(\lambda(p+1)-\Lambda(d+1)) \\
& \geq p|x|^{-p-2} \quad \text { if } p \text { is large enough. }
\end{aligned}
$$

Thus, the function $b(x)=|x|^{-p}$ is a sub-solution of the Pucci equation $M^{-}\left(D^{2} b, \nabla b\right) \geq 0$ in $B_{2} \backslash\{0\}$ with $\gamma=0$. Likewise, it will be a sub-solution of $M^{-}\left(D^{2} b, \nabla b\right) \geq 0$ in $B_{2} \backslash\{0\}$ provided that $\gamma$ is chosen smaller than the minimum norm of its gradient.

Using this barrier function, we prove the following doubling property for lower bounds of super-solutions.

Lemma 4.1 (Doubling property for super-solutions). There exists a small constant $\varepsilon_{0}>0$ depending on $\lambda, \Lambda$ and dimension such that if $u \geq 0$ is a super-solution $M^{-}\left(D^{2} u, \nabla u\right) \leq 1$ in $B_{2}$ and $u>M$ in $B_{1 / 4}$ for some large constant $M$, then $u>1$ in $B_{1}$.

Remark 4.2. The constant $M$ depends on $\lambda, \Lambda, \gamma$ and dimension.

Proof. We compare the function $u$ with

$$
B(x):=M \frac{\left(|x|^{-p}-2^{-p}\right)}{2 \cdot 4^{p}} .
$$

We choose $M \geq 1$ sufficiently large so that $B \geq 1$ in $B_{1}$ and $|\nabla B| \geq \gamma$ in $B_{1}$.

We have

$$
\begin{aligned}
M^{-}\left(D^{2} B, \nabla B\right) & \geq \frac{M}{2 \cdot 4^{p}} M^{-}\left(D^{2} b, \nabla b\right) \\
& \geq \frac{M}{2 \cdot 4^{p}} p 2^{-p-2} \\
& \geq 2 \quad \text { for } M \text { large enough. }
\end{aligned}
$$

Moreover, $B=0$ on $\partial B_{2}$ and $B<M$ in $\partial B_{1 / 4}$. Therefore $B \leq u$ in the ring $B_{2} \backslash B_{1 / 4}$ (this is the comparison principle between the viscosity super-solution $u$ and the classical sub-solution $B$, which follows directly from the definition of viscosity solution).

Therefore, we have $u \geq B \geq 1$ in $B_{1}$. Moreover, for $\varepsilon=\min _{B_{1 / 4}}(u / M-1)$ we also have $u \geq(1+\varepsilon) M>1$ in $B_{1}$, which finishes the proof.

Combining Lemmas 3.1 with 4.1, we obtain the following corollary

Corollary 4.3. There exist small constants $\varepsilon_{0}>0$ and $\delta>0$, and a large constant $M>0$, so that if $\gamma \leq \varepsilon_{0}$, for any continuous function $u: B_{2} \rightarrow \mathbb{R}$ such that

$$
\begin{aligned}
& u \geq 0 \text { in } B_{2}, \\
& M^{-}\left(D^{2} u, \nabla u\right) \leq 1 \text { in } B_{2}, \\
& \left|\{u>M\} \cap B_{1}\right|>(1-\delta)\left|B_{1}\right|,
\end{aligned}
$$

then $u>1$ in $B_{1}$.

Remark 4.4. Note that the constant $M$ in Corollary 4.3 is the product of the two constants $M$ in Lemma 3.1 and Lemma 4.1

Proof. Let $M_{1}$ be the constant from Lemma 3.1 and $M_{2}$ be the one coming from Lemma 4.1 Then the function $v=u / M_{2}$ satisfies the assumption of Lemma 3.1 for $M_{2} \geq 1$ (which can be assumed without loss of generality). We conclude that $v>1$ in $B_{1 / 4}$, i.e. $u>M_{2}$ in $B_{1 / 4}$. We then can apply Lemma 4.1 and get $u>1$ in $B_{1}$. 
The following corollary is just a scaled version of the above result.

Corollary 4.5. There exists small constants $\varepsilon_{0}>0$ and $\delta>0$, and a large constant $M>0$, so that if $\gamma \leq \varepsilon_{0}$, for any $r \leq 1, \kappa \geq 1$ and a continuous function $u: \overline{B_{r}} \rightarrow \mathbb{R}$ such that

$$
\begin{aligned}
& u \geq 0 \text { in } B_{r}, \\
& M^{-}\left(D^{2} u, \nabla u\right) \leq \kappa \text { in } B_{r}, \\
& \left|\{u>\kappa M\} \cap B_{r / 2}\right|>(1-\delta)\left|B_{r / 2}\right|,
\end{aligned}
$$

then $u>\kappa$ in $B_{r / 2}$.

Proof. The scaled function $u_{r}(x)=u(r x / 2) / \kappa$ satisfies the scaled equation

$$
M_{r / 2, \kappa}^{-}\left(D^{2} u_{r}, \nabla u_{r}\right) \leq r^{2} \leq 1 \text { in } B_{2}
$$

We remark that it satisfies a stronger equation since $\gamma$ can be replaced by the smaller value $\kappa^{-1} r \gamma$. So we can apply Corollary 4.3 to $u_{r}$ and obtain the result.

\section{The $L^{\varepsilon}$ estimate}

Combining Corollary 4.3 with Lemma 2.1. we obtain the $L^{\varepsilon}$ estimate.

Theorem 5.1 ( $L^{\varepsilon}$ estimate). There exists small constants $\varepsilon_{0}>0$ and $\varepsilon>0$, so that if $\gamma \leq \varepsilon_{0}$, for any lower semi-continuous function $u: B_{2} \rightarrow \mathbb{R}$ such that

$$
\begin{aligned}
& u \geq 0 \text { in } B_{2}, \\
& M^{-}\left(D^{2} u, \nabla u\right) \leq 1 \text { in } B_{2}, \\
& \inf _{B_{1}} u \leq 1,
\end{aligned}
$$

then

$$
\left|\{u>t\} \cap B_{1}\right| \leq C t^{-\varepsilon}
$$

for all $t>0$.

Remark 5.2. This estimate is referred to as the $L^{\varepsilon}$ estimate since it yields an estimate on $\int_{B_{1}} u^{\varepsilon}(x) d x$ (depending on $C$ only).

Proof. In order to prove the result, we will prove the equivalent expression

$$
\left|\left\{u>M^{k}\right\} \cap B_{1}\right| \leq \tilde{C} M^{-\varepsilon k}
$$

where $M$ is the constant from Corollary 4.5 and $\varepsilon>0$ has to be properly chosen.

Let $A_{k}:=\left\{u>M^{k}\right\} \cap B_{1}$, which are open sets. Since $\inf _{B_{1}} u \leq 1$, from Corollary 4.3 . $\left|A_{1}\right| \leq(1-\delta)\left|B_{1}\right|$. Since $A_{k} \subset A_{1}$ for all $k>1$, then we also have $\left|A_{k}\right| \leq(1-\delta)\left|B_{1}\right|$ for all $k$.

We note that Corollary 4.5, with $\kappa=M^{k}$, says that every time a ball $B \subset B_{1}$ satisfies $\left|B \cap A_{k+1}\right|>$ $(1-\delta)|B|$, then $B \subset A_{k}$. Using Lemma2.1, we obtain

$$
\left|A_{k+1}\right| \leq(1-c \delta)\left|A_{k}\right|
$$

and therefore, by induction, $\left|A_{k}\right| \leq(1-c \delta)^{k-1}(1-\delta)\left|B_{1}\right|=\tilde{C} M^{-\varepsilon k}$, where $-\varepsilon=\log (1-c \delta) / \log M$ and $\tilde{C}=(1-c \delta)^{-1}(1-\delta)\left|B_{1}\right|$.

This finishes the proof.

The following lemma is a scaled version of Theorem 5.1 
Lemma 5.3 (scaled $L^{\varepsilon}$ estimate). There exists small constants $\tilde{\varepsilon}_{0}>0, \varepsilon_{1}>0$ and $\theta>0$, so that if $\gamma \leq \tilde{\varepsilon}_{0}$, for any $r \leq 1, \alpha \in(0,1)$, and a lower semi-continuous function $u: \overline{B_{2 r}} \rightarrow \mathbb{R}$ such that

$$
\begin{aligned}
& u \geq 0 \text { in } B_{2 r}, \\
& M^{-}\left(D^{2} u, \nabla u\right) \leq \varepsilon_{1} \text { in } B_{2 r}, \\
& \left|\left\{u>r^{\alpha}\right\} \cap B_{r}\right| \geq \frac{1}{2}\left|B_{r}\right|,
\end{aligned}
$$

then $u>\varepsilon_{1} r^{\alpha}$ in $B_{r}$.

Remark 5.4. As we shall see when proving this lemma, $\tilde{\varepsilon}_{0}=\varepsilon_{0} \varepsilon_{1}$ where $\varepsilon_{0}$ is given by Lemma 5.1

Proof. Let $\tau$ be the universal constant such that $C \tau^{-\varepsilon}<\left|B_{1}\right| / 2$, where $C$ and $\varepsilon$ are the constants of Theorem 5.1. Consider the function $\tilde{u}(x)=\tau r^{-\alpha} u(r x)$. It satisfies the properties

$$
\begin{aligned}
& \tilde{u} \geq 0 \text { in } B_{2}, \\
& M^{-}\left(D^{2} \tilde{u}, \nabla \tilde{u}\right) \leq \tau r^{2-\alpha} \varepsilon_{1} \text { in } B_{2}, \\
& \left|\{\tilde{u}>\tau\} \cap B_{1}\right| \geq \frac{1}{2}\left|B_{1}\right|>C \tau^{-\varepsilon},
\end{aligned}
$$

with $\tau r^{1-\alpha} \gamma$ instead of $\gamma$.

Let us choose $\varepsilon_{1}=\tau^{-1}$. Since $r \leq 1$, we have

$$
M^{-}\left(D^{2} \tilde{u}, \nabla \tilde{u}\right) \leq 1 \text { in } B_{2} .
$$

We now apply Theorem 5.1 and obtain that $\tilde{u}>1$ in $B_{1}$ provided that $\tau r^{1-\alpha} \gamma \leq \varepsilon_{0}$. We just have to choose $\tilde{\varepsilon}_{0}=\varepsilon_{0} \tau^{-1}=\varepsilon_{0} \varepsilon_{1}$ since $r^{1-\alpha} \leq 1$. Scaling back, we obtain $u>\varepsilon_{1} r^{\alpha}$ in $B_{r}$.

\section{Hölder continuity}

In this section, we derive the Hölder estimates of Theorem 1.1 from the (scaled) $L^{\varepsilon}$ estimate.

Proof of Theorem 1.1. We start by normalizing the solution $u$. Let

$$
v(x)=\frac{u(\rho x)}{C_{0}\left(1+\varepsilon_{1}^{-1}\right)},
$$

where $\rho \leq 1$ and $\varepsilon_{1}$ is the constant from Lemma 5.3. The function $v$ satisfies the estimates

$$
\begin{aligned}
& M^{-}\left(D^{2} v, \nabla v\right) \leq \varepsilon_{1} \text { in } B_{1}, \\
& M^{+}\left(D^{2} v, \nabla v\right) \geq-\varepsilon_{1} \text { in } B_{1}, \\
& \|v\|_{L^{\infty}\left(B_{1}\right)} \leq 1
\end{aligned}
$$

with $\gamma$ replaced by $\frac{\rho}{C_{0}\left(1+\varepsilon_{1}^{-1}\right)} \gamma$. Thus, we pick $\rho \leq 1$ such that

$$
\frac{\rho \gamma}{C_{0}\left(1+\varepsilon_{1}^{-1}\right)} \leq \tilde{\varepsilon}_{0}
$$

where $\tilde{\varepsilon}_{0}$ is given by Lemma 5.3. It is enough to pick

$$
\rho=\min \left(1, \frac{\tilde{\varepsilon}_{0} C_{0}\left(1+\varepsilon_{1}^{-1}\right)}{\gamma}\right) .
$$

Let $a_{k}=\min _{B_{2^{-k}}} v$ and $b_{k}=\max _{B_{2^{-k}}} v$. We will prove that for some $\alpha>0$,

$$
b_{k}-a_{k} \leq 2 \times 2^{-\alpha k} .
$$


For $k=0$, the statement is obvious since $b_{0} \leq\|v\|_{L^{\infty}\left(B_{1}\right)}$ and $a_{0} \geq-\|v\|_{L^{\infty}\left(B_{1}\right)}$, thus $b_{0}-a_{0} \leq 2$. Now we proceed by induction.

Assume that $b_{k}-a_{k} \leq 2 \times 2^{-\alpha k}$ and let us prove that $b_{k+1}-a_{k+1} \leq 2 \times 2^{-\alpha(k+1)}$. If $b_{k}-a_{k} \leq 2 \times 2^{-\alpha(k+1)}$, then we are done since $b_{k+1}-a_{k+1} \leq b_{k}-a_{k}$. Hence, we can assume that $\frac{b_{k}-a_{k}}{2} \geq 2^{-\alpha(k+1)}$.

Let $m_{k}=\left(a_{k}+b_{k}\right) / 2$. We have two alternatives. Either $\left|\left\{v>m_{k}\right\} \cap B_{2^{-k-1}}\right| \geq\left|B_{2^{-k-1}}\right| / 2$ or $\mid\{v \leq$ $\left.m_{k}\right\} \cap B_{2^{-k-1}}|\geq| B_{2^{-k-1}} \mid / 2$. In the first case we will prove that $a_{k+1}$ is larger than $a_{k}$, whereas in the second case we will show that $b_{k+1}$ is smaller than $b_{k}$.

Let us assume the first case, i.e. $\left|\left\{v>m_{k}\right\} \cap B_{2^{-k-1}}\right| \geq\left|B_{2^{-k-1}}\right| / 2$. We apply Lemma 5.3 to $v-a_{k}$ with $r=2^{-k-1}$ to obtain that $v-a_{k} \geq \varepsilon_{1} 2^{-(k+1) \alpha}$ for some $\varepsilon_{1}>0$ universal. Therefore, we have that $a_{k+1} \geq a_{k}+\varepsilon_{1} 2^{-(k+1) \alpha}$. In particular

$$
b_{k+1}-a_{k+1} \leq b_{k}-a_{k}-\varepsilon_{1} 2^{-(k+1) \alpha} \leq\left(2^{\alpha+1}-\varepsilon_{1}\right) 2^{-(k+1) \alpha} \leq 2 \times 2^{-(k+1) \alpha}
$$

as soon as $\alpha$ is chosen small enough so that $2^{\alpha+1} \leq 2+\varepsilon_{1}$.

The estimate (6.1) implies that $v$ is $C^{\alpha}$ at the origin, with

$$
|v(x)-v(0)| \leq 4|x|^{\alpha},
$$

for all $x \in B_{1}$. Scaling back to the function $u$, this means that for all $x \in B_{\rho}$,

$$
\begin{aligned}
|u(x)-u(0)| & \leq 4\left(1+\varepsilon_{1}^{-1}\right) \rho^{-\alpha} C_{0}|x|^{\alpha} \\
& \leq C C_{0}|x|^{\alpha}
\end{aligned}
$$

where $C=C\left(\lambda, \Lambda, d, \gamma / C_{0}\right)$. By a standard translation and covering argument, we have that $u \in C^{\alpha}\left(B_{1 / 2}\right)$ and

$$
[u]_{C^{0, \alpha}\left(B_{1 / 2}\right)} \leq \tilde{C} C_{0}
$$

where $\tilde{C}$ differs from $C$ by a universal constant The proof is now complete.

\section{Harnack inequality}

This section is devoted to the derivation of a Harnack inequality.

Proof of Theorem 1.3. We first reduce the problem to $C_{0}=1$ and $\inf _{B_{1 / 2}} u \leq 1$ by replacing $u$ with $u /\left(C_{0}+\right.$ $\left.\inf _{B_{1 / 2}} u\right)$. In particular, $\gamma$ is replaced with $\gamma /\left(C_{0}+\inf _{B_{1 / 2}} u\right)$.

Let $\beta>0$ and let $h_{t}(x)=t(3 / 4-|x|)^{-\beta}$ be defined in $B_{3 / 4}$. We consider the minimum value of $t$ such that $h_{t} \geq u$ in $B_{3 / 4}$. The objective of the proof is to show that this value of $t$ cannot be too large. If $t \leq 1$, we are done. Hence, we further assume that $t \geq 1$.

Since $t$ is chosen to be the minimum value such that $h_{t} \geq u$, then there must exist some $x_{0} \in B_{1}$ such that $h_{t}\left(x_{0}\right)=u\left(x_{0}\right)$. Let $r=\left(3 / 4-\left|x_{0}\right|\right) / 2$. That is, $2 r$ is the distance from $x_{0}$ and $\partial B_{3 / 4}$. Let $H_{0}:=h_{t}\left(x_{0}\right)=t(2 r)^{-\beta} \geq 1$.

We will estimate the measure of the set $\left\{u \geq H_{0} / 2\right\} \cap B_{r}\left(x_{0}\right)$ in two different ways. We will get a contradiction if $t$ is too large.

Let us start by an upper bound of the measure. From Theorem 5.1 properly rescaled,

$$
\left|\left\{u>H_{0}\right\} \cap B_{r}\left(x_{0}\right)\right| \leq\left|\left\{u>H_{0}\right\} \cap B_{3 / 4}\right| \leq H_{0}^{-\varepsilon}=C t^{-\varepsilon}(2 r)^{\beta \varepsilon} .
$$

Let us now obtain a lower bound. Let $\mu$ be the small universal constant and $\beta$ be a large universal 
constant such that

$$
\begin{aligned}
M\left(\left(\frac{2-\mu}{2}\right)^{-\beta}-1\right) & \leq \frac{1}{2} \\
\frac{(\mu r)^{2}}{\left(\left(\frac{2-\mu}{2}\right)^{-\beta}-1\right)} & \leq 1, \\
\frac{(\mu r) \gamma}{\left(\left(\frac{2-\mu}{2}\right)^{-\beta}-1\right)} & \leq \varepsilon_{0} \\
\beta & \geq \frac{d}{\varepsilon} .
\end{aligned}
$$

where $M$ and $\varepsilon_{0}$ are constants from Corollary 4.3 and $\varepsilon$ comes from Theorem 5.1 . The reader can check that choosing

$$
\beta=\varepsilon^{-1} \max (d, \gamma)
$$

and $\mu$ small enough so that

$$
\mu \leq \frac{\gamma}{\varepsilon_{0}} \quad \text { and } \quad \frac{\ln (1+\mu \gamma / \varepsilon)}{-\ln (1-\mu / 2)} \leq \frac{\gamma}{\varepsilon} \quad \text { and } \quad(1-\mu / 2)^{-\beta}-1 \leq \frac{1}{2 M}
$$

we get the four desired inequalities.

The maximum of $u$ in the ball $B_{\mu r}\left(x_{0}\right)$ is at most the maximum of $h_{t}$ which equals $t(2 r-\mu r)^{-\beta}=$ $\left(\frac{2-\mu}{2}\right)^{-\beta} H_{0}$. Let us define the function

$$
v(x)=\frac{\left(\frac{2-\mu}{2}\right)^{-\beta} H_{0}-u\left(x_{0}+\mu r x\right)}{\left(\left(\frac{2-\mu}{2}\right)^{-\beta}-1\right) H_{0}} .
$$

Note that $v(0)=1, v$ is non-negative in $B_{1}$ and satisfies the equations

$$
\begin{aligned}
& M^{-}\left(D^{2} v, \nabla v\right) \leq \frac{(\mu r)^{2}}{\left(\left(\frac{2-\mu}{2}\right)^{-\beta}-1\right) H_{0}} \leq 1 \text { in } B_{1} \\
& M^{+}\left(D^{2} v, \nabla v\right) \geq-\frac{(\mu r)^{2}}{\left(\left(\frac{2-\mu}{2}\right)^{-\beta}-1\right) H_{0}} \geq-1 \text { in } B_{1}
\end{aligned}
$$

with $\gamma$ replaced by $\frac{(\mu r) \gamma}{H_{0}\left(\left(\frac{2-\mu}{2}\right)^{-\beta}-1\right)} \leq \varepsilon_{0}$ (because of the choice of $\mu$ and $\beta$ ).

We can apply Corollary 4.3 (in fact, its counterpositive) and obtain

$$
\left|\{v \leq M\} \cap B_{1 / 2}\right| \geq \delta\left|B_{1 / 2}\right|
$$

In terms of the original function $u$, this is an estimate of a set where $u$ is larger than

$$
H_{0}\left(\left(\frac{2-\mu}{2}\right)^{-\beta}-M\left(\left(\frac{2-\mu}{2}\right)^{-\beta}-1\right)\right) \geq \frac{H_{0}}{2},
$$

because of the choice of $\mu$ and $\beta$. Thus, we obtain the estimate

$$
\left|\left\{u \geq H_{0} / 2\right\} \cap B_{\mu r}\left(x_{0}\right)\right| \geq \delta\left|B_{\mu r}\right|
$$

Together with (7.1), this implies that $t$ is bounded from above (using the fact that $\beta \geq d / \varepsilon$ ). 


\section{Acknowledgments}

Cyril Imbert was partially supported by ANR grant ANR-12-BS01-0008-01. Luis Silvestre was partially supported by NSF grants DMS-1254332 and DMS-1065979.

\section{References}

[1] Roberto Argiolas, Fernando Charro, and Ireneo Peral. On the Aleksandrov-Bakel'man-Pucci estimate for some elliptic and parabolic nonlinear operators. Arch. Ration. Mech. Anal., 202(3):875-917, 2011.

[2] S. N. Armstrong and C. K. Smart. Regularity and stochastic homogenization of fully nonlinear equations without uniform ellipticity. Ann. Probab. (to appear), 2012. arXiv:1208.4570.

[3] I. Birindelli and F. Demengel. Eigenfunctions for singular fully nonlinear equations in unbounded domains. NoDEA Nonlinear Differential Equations Appl., 17(6):697-714, 2010.

[4] Xavier Cabré. Nondivergent elliptic equations on manifolds with nonnegative curvature. Communications on pure and applied mathematics, 50(7):623-665, 1997.

[5] Luis A. Caffarelli and Xavier Cabré. Fully nonlinear elliptic equations, volume 43 of American Mathematical Society Colloquium Publications. American Mathematical Society, Providence, RI, 1995.

[6] Fernando Charro, Guido De Philippis, Agnese Di Castro, and Davi Máximo. On the AleksandrovBakelmanPucci estimate for the infinity Laplacian. Calculus of Variations and Partial Differential Equations, pages $1-27,2012$.

[7] Gonzalo Dávila, Patricio Felmer, and Alexander Quaas. Alexandroff-Bakelman-Pucci estimate for singular or degenerate fully nonlinear elliptic equations. C. R. Math. Acad. Sci. Paris, 347(19-20):1165-1168, 2009.

[8] Gonzalo Dávila, Patricio Felmer, and Alexander Quaas. Harnack inequality for singular fully nonlinear operators and some existence results. Calc. Var. Partial Differential Equations, 39(3-4):557-578, 2010.

[9] François Delarue. Krylov and Safonov estimates for degenerate quasilinear elliptic PDEs. J. Differential Equations, 248(4):924-951, 2010.

[10] J.-B. Hiriart-Urruty. The approximate first-order and second-order directional derivatives for a convex function. In Mathematical theories of optimization (Genova, 1981), volume 979 of Lecture Notes in Math., pages 144-177. Springer, Berlin, 1983.

[11] Cyril Imbert. Alexandroff-Bakelman-Pucci estimate and Harnack inequality for degenerate/singular fully non-linear elliptic equations. J. Differential Equations, 250(3):1553-1574, 2011.

[12] T. Junges Miotto. The Aleksandrov-Bakelman-Pucci estimates for singular fully nonlinear operators. Commun. Contemp. Math., 12(4):607-627, 2010.

[13] N. V. Krylov and M. V. Safonov. An estimate for the probability of a diffusion process hitting a set of positive measure. Dokl. Akad. Nauk SSSR., 245 (1): 18-20, 1979.

[14] N. V. Krylov and M. V. Safonov. A property of the solutions of parabolic equations with measurable coefficients. Izv. Akad. Nauk SSSR Ser. Mat., 44 (1):161-175, 1980.

[15] M. V. Safonov. Non-divergence elliptic equations of second order with unbounded drift. In Nonlinear partial differential equations and related topics, volume 229 of Amer. Math. Soc. Transl. Ser. 2, pages 211-232. Amer. Math. Soc., Providence, RI, 2010.

[16] Ovidiu Savin. Small perturbation solutions for elliptic equations. Comm. Partial Differential Equations, 32(4-6):557-578, 2007. 